\title{
Stabilizing Lithium Metal Anode by Ion Depletion-Induced Phase Transformation in Polymer Electrolytes
}

Qian Cheng, ${ }^{1,6}$ Yupeng Miao, ${ }^{2,6}$ Zhe Liu, ${ }^{3}$ James Borovilas, ${ }^{1}$ Hanrui Zhang, ${ }^{1}$ Shuwei Liu, ${ }^{1}$ Haozhen Wang, ${ }^{4}$ Xi Chen,${ }^{4,5}$ Long-Qing Chen, ${ }^{3}$ Wei Min,${ }^{2 *}$ Yuan Yang ${ }^{1 *}$

${ }^{1}$ Program of Materials Science and Engineering, Department of Applied Physics and Applied Mathematics, Columbia University, New York, NY 10027, USA

${ }^{2}$ Department of Chemistry, Columbia University, New York, NY, 10027, USA

${ }^{3}$ Department of Materials Science and Engineering, The Pennsylvania State University, University Park, State College, PA 16802, USA.

${ }^{4}$ Advanced Science Research Center (ASRC), City University of New York, 85 St. Nicholas Terrace, New York, NY, 10031 USA

${ }^{5}$ Department of Chemical Engineering, The City College of New York, 160 Convent Avenue, New York, NY 10031, USA

${ }^{6}$ These authors contributed equally

Correspondence: Y.Y. (yy2664@ columbia.edu) or W.M. (wm2256@ columbia.edu)

\begin{abstract}
Ion depletion in liquid electrolytes is widely accepted to promote dendrite growth in metal anodes due to enhanced local electrical field and magnified concentration fluctuation at the electrode/electrolyte interface. Here we report unexpected opposite behaviors in solid polymer electrolytes, showing that ion depletion leads to uniform lithium deposition. Such stabilization originates from ion depletion-induced phase transformation, which forms a new PEO-rich but salt/plasticizer-poor phase at the lithium/electrolyte interface, as unveiled by stimulated Raman scattering microscopy. This new phase leads a significantly higher Young's modulus ( 2-3 GPa) than the bulk polymer electrolyte $(<10 \mathrm{MPa})$, which effectively suppresses dendrite growth. Further battery tests show that $\mathrm{LiFePO}_{4} / \mathrm{PEO} / \mathrm{Li}$ cells with such ion depletion-induced phase transformations can be reversibly cycled for 200 times, while cells without such transformation fail within only ten cycles, demonstrating the effectiveness of this strategy to stabilize the lithium anode.
\end{abstract}




\section{Introduction}

Lithium-metal batteries (LMBs) have attracted significant attention in recent years for next-generation energy storage with high energy density ${ }^{1,2,3}$. Li metal anode has a theoretical capacity of $3860 \mathrm{mAh} \mathrm{g}^{-1}$, ten times that of the conventional graphite anode, and an ultralow electrode potential (-3.04 V vs. standard hydrogen electrode / SHE). However, lithium deposition is prone to being non-uniform, leading to rough morphologies such as mossy and dendritic lithium ${ }^{4}$, 5,6,7. Such uneven deposition not only results in a large electrode surface area which promotes side reactions with electrolyte and decreases coulombic efficiency and cycle life ${ }^{8,9}$, but also imposes potential safety hazards such as internal shorting and thermal runaway, especially in combination with conventional flammable liquid electrolytes (e.g. ethers and carbonates) ${ }^{10,11,12,13}$.

Polymer electrolytes are appealing for enhancing the thermal stability of LMBs as they are much more thermally stable than liquid electrolytes ${ }^{14,15,16}$. For example, the flash points (f.p.) of poly (ethylene oxide) is $250{ }^{\circ} \mathrm{C}{ }^{17}$, much higher than dimethyl carbonate $\left(21.5^{\circ} \mathrm{C}\right)^{18}, 1,3$-dioxolane $\left(2{ }^{\circ} \mathrm{C}\right)^{19}$ and 1,2-dimethoxyethane $\left(-2{ }^{\circ} \mathrm{C}\right)^{17}$ in liquid electrolytes. Polymer electrolytes are also compatible with conventional battery manufacturing processes and easy to scale up ${ }^{20}$. Unfortunately, Young's moduli of PEO-based polymer electrolytes are typically in the range of 20 to $70 \mathrm{MPa}{ }^{21,22,23}$, much lower than the proposed threshold of $1 \mathrm{GPa}$ needed to suppress $\mathrm{Li}$ dendrites ${ }^{11,24}$. Hence, the fast growth of Li dendrites in polymer electrolytes is widely observed in literature 24, 25, 26, and Supplementary Movie 1. The dendrite growth becomes even more severe with the introduction of plasticizers for enhancing ionic conductivity, as they further soften the electrolyte $^{23,27}$. This issue is difficult to be fully resolved by ceramic additives, ${ }^{28}$ since lithium dendrites can still penetrate through the interspace between ceramic fillers. Currently, Li dendrite growth remains one of the major challenges in polymer electrolyte-based LMBs. ${ }^{29}$

Addressing this issue requires fundamental understanding of the embedded dynamic $\mathrm{Li}$ metal/polymer electrolyte interface, such as how $\mathrm{Li}^{+}$heterogeneity evolves at the $\mathrm{Li}$ anode surface and how the Li anode interacts with solid electrolytes. While remarkable advances have been achieved recently in characterizing the Li anode and solid electrolyte interphase (SEI) in liquid electrolytes, such as cryo-transmission electron microscope (TEM), ${ }^{30,31,32}$ nuclear magnetic resonance (NMR), ${ }^{33,34}$ in-situ and environmental TEM, ${ }^{35,36}$ synchrotron, ${ }^{37,38}$ and ambient pressure $\mathrm{XPS}^{39}$, there is limited progress towards imaging the interaction between $\mathrm{Li}^{+}$transport in the 
electrolyte and Li dendrite growth. This arises from challenges in visualizing ions in the electrolyte, which not only has a low concentration (0.01-2 M), but also possesses fast dynamics (diffusivity of $\left.\sim 10^{-7}-10^{-6} \mathrm{~cm}^{2} \mathrm{~s}^{-1}\right)$ compared to solid electrodes $\left(10-50 \mathrm{M}\right.$, and $\left.<10^{-9} \mathrm{~cm}^{2} \mathrm{~s}^{-1}\right)$. Hence, ultrahigh chemical sensitivity $(\sim 1-10 \mathrm{mM})$, high speed $(\sim 1 \mathrm{~s} / \mathrm{image})$, and fine spatial resolution $(<1 \mu \mathrm{m})$ are simultaneously required to image the electrolyte, especially during ion depletion. Such resolutions and sensitivity are beyond the capability of conventional characterization tools.

Recently we utilized stimulated Raman scattering (SRS) microscopy to image ion transport profile in liquid electrolytes, which simultaneously offers high sensitivity $(<1 \mathrm{mM})$, fast imaging speed ( $\sim 2 \mu$ s per pixel), and fine spatial resolution (down to $300 \mathrm{~nm})^{40,41,42}$. SRS microscopy uses two temporally and spatially synchronized laser beams with an energy difference equal to that of the target bond's vibrational mode. The synergy of the two beams amplifies the otherwise weak vibrational signal by up to $10^{8}$ times. It thus enables the desired resolutions, imaging speed, and sensitivity (Fig. 1a, Supplementary Fig. 1). ${ }^{43}$ Using this emerging microscopy, we visualized for the first time the heterogeneity of ion depletion in liquid electrolytes and how it correlated with lithium dendrite growth. ${ }^{40} \mathrm{We}$ observed that ion depletion at the Li/electrolyte interface led to faster dendrite growth due to the enhanced electrical field and larger concentration heterogeneity, which agreed with theoretical predictions. ${ }^{1,44}$

In this report, we further explored the solid polymer electrolyte/electrode interaction and observed unexpected opposite phenomena. Instead of promoting dendrite formation, ion depletion strongly suppressed dendrite growth in PEO electrolytes and led to reversible lithium plating and stripping. Further analysis unveiled that this unusual behavior originated from an ion depletioninduced phase transformation in the polymer electrolyte and subsequent mechano-chemical coupling inside (Fig. 1b). Once ions deplete, a new PEO-rich phase was formed at the lithium/electrolyte interface with a high modulus 1-3 GPa, as measured by atomic force microscopy (AFM). Such a high modulus suppressed dendrite growth and led to uniform lithium deposition. Based on this discovery, we developed a PEO electrolyte with an optimal composition (EO/Li = 6, 1.6 M Li salt), which showed stable cycling over 200 times in an LFP/PEO/Li cell at $38{ }^{\circ} \mathrm{C}$. In contrast, LFP/PEO/Li cells with concentrated electrolyte (EO/Li = 3, 2.7 M Li salt) failed quickly after 10 cycles due to the rapid growth of lithium dendrites and dramatically 
increased impedance. This work proposes a new strategy for using self-forming mechanically strong phases to stabilize the lithium metal anode in solid-polymer-electrolyte-based LMBs.

a

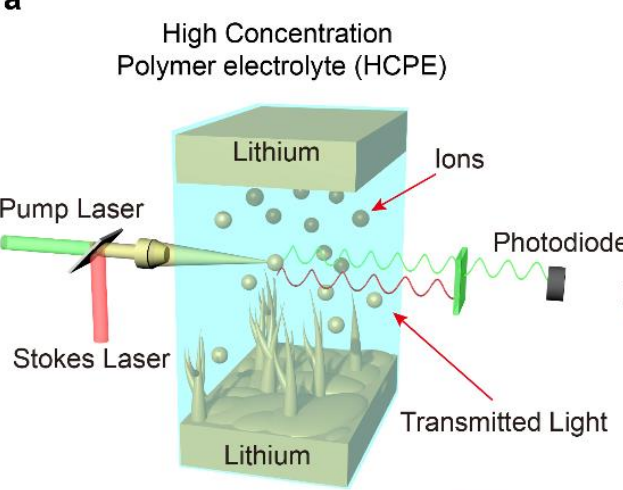

b

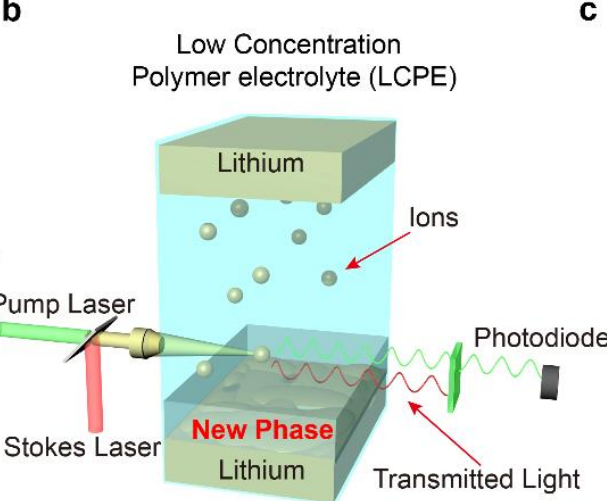

Polymer Electrolyte

d

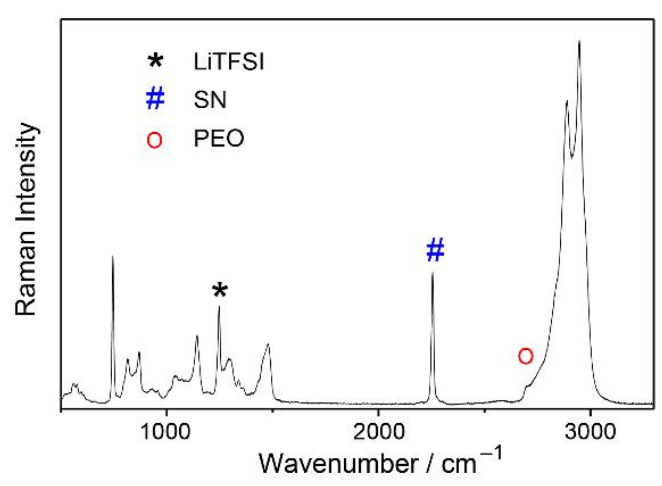

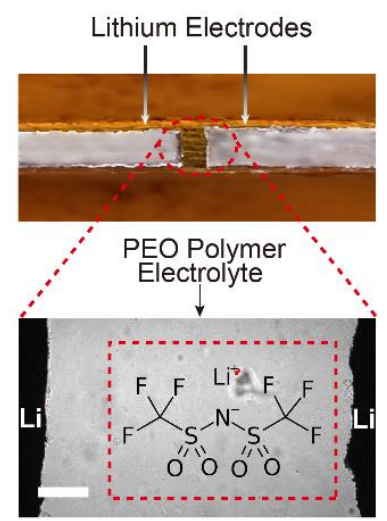

O : Li molar ratio

e

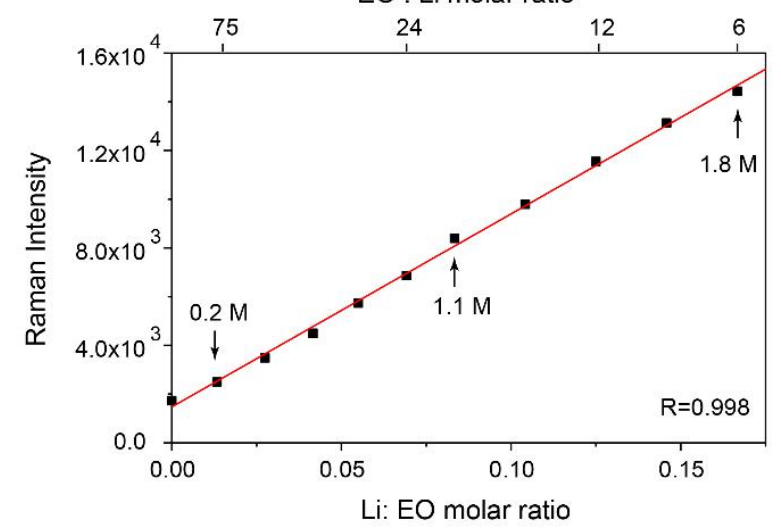

Figure 1. The schematics of operando SRS imaging of the Li/PEO electrolyte interface. The schematics of SRS observations on a, high concentration polymer electrolyte (HCPE) and $\mathbf{b}$, low concentration polymer electrolyte (LCPE) in Li/Li cells. In LCPE, the ion depletion-induced phase transformation leads to a mechanically strong PEO-rich phase at the lithium/electrolyte interface, which helps suppress lithium dendrites. $\mathbf{c}$, the bright field of a $\mathrm{Li} / \mathrm{PEO} / \mathrm{Li}$ cell in operando. The upper picture shows the structure of the cell, while the lower one shows a zoom-in image under the microscope. d, the Raman spectrum of LCPE, where signature peaks for LiTFSI, SN, and PEO are labeled. The corresponding composition of LCPE is EO: Li: $\mathrm{SN}=12: 1: 2.64$. e, the plot of Raman intensity of the LiTFSI peak at $1245 \mathrm{~cm}^{-1}$ versus the Li: EO ratio in PEO electrolytes with $40 \mathrm{wt} \% \mathrm{SN}$, showing good linearity. The points of $1.1 \mathrm{M}$ and $1.8 \mathrm{M}$ LiTFSI correspond to LCPE and HCPE, respectively. 


\section{Results}

SRS imaging of $\mathbf{L i}$ / solid polymer electrolyte interaction. Home-made parallel cells were used to visualize the Li / PEO electrolyte interaction via SRS microscopy (Fig. 1c). In this cell, the PEO electrolyte filled in the gap between two pieces of lithium, and all components were sandwiched between two glass slides and sealed by epoxy. The distance between two electrodes was typically $\sim 0.5 \mathrm{~mm}$. The PEO electrolyte contained lithium bis(trifluoromethanesulfonyl) imide (LiTFSI) as the salt and succinonitrile (SN) as the plasticizer ${ }^{45}$ to enhance ionic conductivity and enable operation at room temperature. Wavenumbers at $1245 \mathrm{~cm}^{-1}\left(\mathrm{CF}_{3}\right.$ stretching $),{ }^{46} 2250 \mathrm{~cm}^{-1}(\mathrm{C} \equiv \mathrm{N}$ stretching), ${ }^{47}$ and $2800 \mathrm{~cm}^{-1}(\mathrm{C}-\mathrm{H} \text { stretching with combination vibration })^{48}$ were selected for SRS imaging of LiTFSI, SN and PEO, respectively (Fig. 1d). Due to the requirement of electroneutrality, $\left[\mathrm{Li}^{+}\right]$could be considered to be equal to $\left[\mathrm{TFSI}^{-}\right]$, even at the nanoscale (Supplementary Note 2), with an error smaller than $0.1 \mathrm{mM}^{40}$ Therefore, [TFSI ${ }^{-}$] was measured to represent the local $\left[\mathrm{Li}^{+}\right]$. The Raman intensity of $\mathrm{TFSI}^{-}$is proportional to its concentration. Hence the Raman signal can be easily converted to chemical concentrations (Fig. 1e). The chemical sensitivity of LiTFSI was calculated to be $0.012 \mathrm{M}$.

Lithium growth in PEO electrolytes with high and low concentration. As two representatives, the high-concentration polymer electrolyte (HCPE) and the low-concentration polymer electrolyte (LCPE) were studied with EO: Li: $\mathrm{SN}=12: 2: 2.64$ and 12: 1: 2.64 in molarity, respectively, which corresponded to 1.8 M LiTFSI and 2.4 M SN for HCPE, and 1.1 M LiTFSI and 2.9 M SN for LCPE. The weight ratios of SN to PEO were fixed at $40 \%$ in both cases. Their ionic conductivities are $1.7 \times 10^{-4} \mathrm{~S} \mathrm{~cm}^{-1}(\mathrm{HCPE})$ and $1.0 \times 10^{-4} \mathrm{~S} \mathrm{~cm}^{-1}$ (LCPE) at room temperature (RT), respectively (Supplementary Fig. 2). Due to the relatively low ionic conductivities at RT, a current density of $0.5 \mathrm{~mA} \mathrm{~cm}^{-2}$ was applied.

In the case of HCPE (Fig. 2a \& Supplementary Movie 2), the applied current gradually depleted $\left[\mathrm{Li}^{+}\right]$on the lithium surface $\left(\left[\mathrm{Li}^{+}\right]_{0} \mu \mathrm{m}\right)$ from $1.8 \mathrm{M}$ at $\mathrm{t}=0$ to $1.2 \mathrm{M}$ at $\mathrm{t}=27 \mathrm{~min}$, after which $\left[\mathrm{Li}^{+}\right]_{0 \mu \mathrm{m}}$ remained at $\sim 1.2 \mathrm{M}$ in a steady state (Fig. 2b \& c). Meanwhile, the lithium growth rate $(v)$ quickly increased from $0.27 \pm 0.18 \mu \mathrm{m} \mathrm{min}^{-1}$ at $\mathrm{t}=0$ to $0.9 \pm 0.46 \mu \mathrm{m} \mathrm{min}^{-1}$ at $\mathrm{t}=27 \mathrm{~min}$ (Fig. 2d). The growth was in the form of mossy lithium, and statistical analysis showed a normal distribution of $v$ (Supplementary Fig. 3a-c). Afterwards, $v$ was drastically increased to $\sim 1.5 \mu \mathrm{m}$ $\mathrm{min}^{-1}$ for the remaining time, leading to an ultrahigh porosity of $97 \%$, indicating that the HCPE 
could not suppress dendrite growth at all. A dual-peak normal distribution was observed at this stage (Supplementary Fig. 3d-h), where $v$ was low in part of the surface $\left(<1 \mu \mathrm{m} \mathrm{min}{ }^{-1}\right.$ ), but ultrahigh in other regions ( $>2 \mu \mathrm{m} \mathrm{min}^{-1}$ ), showing the heterogeneous dendrite growth on lithium electrode. This dual-peak mode may arise from SEI properties and non-uniform depletion of ions. These results shows that if no phase transformation occurs, ion depletion at the Li/polymer electrolyte interface promotes dendrite growth (Supplementary Movie 2), similar to our previous observations in gel electrolyte. ${ }^{40}$
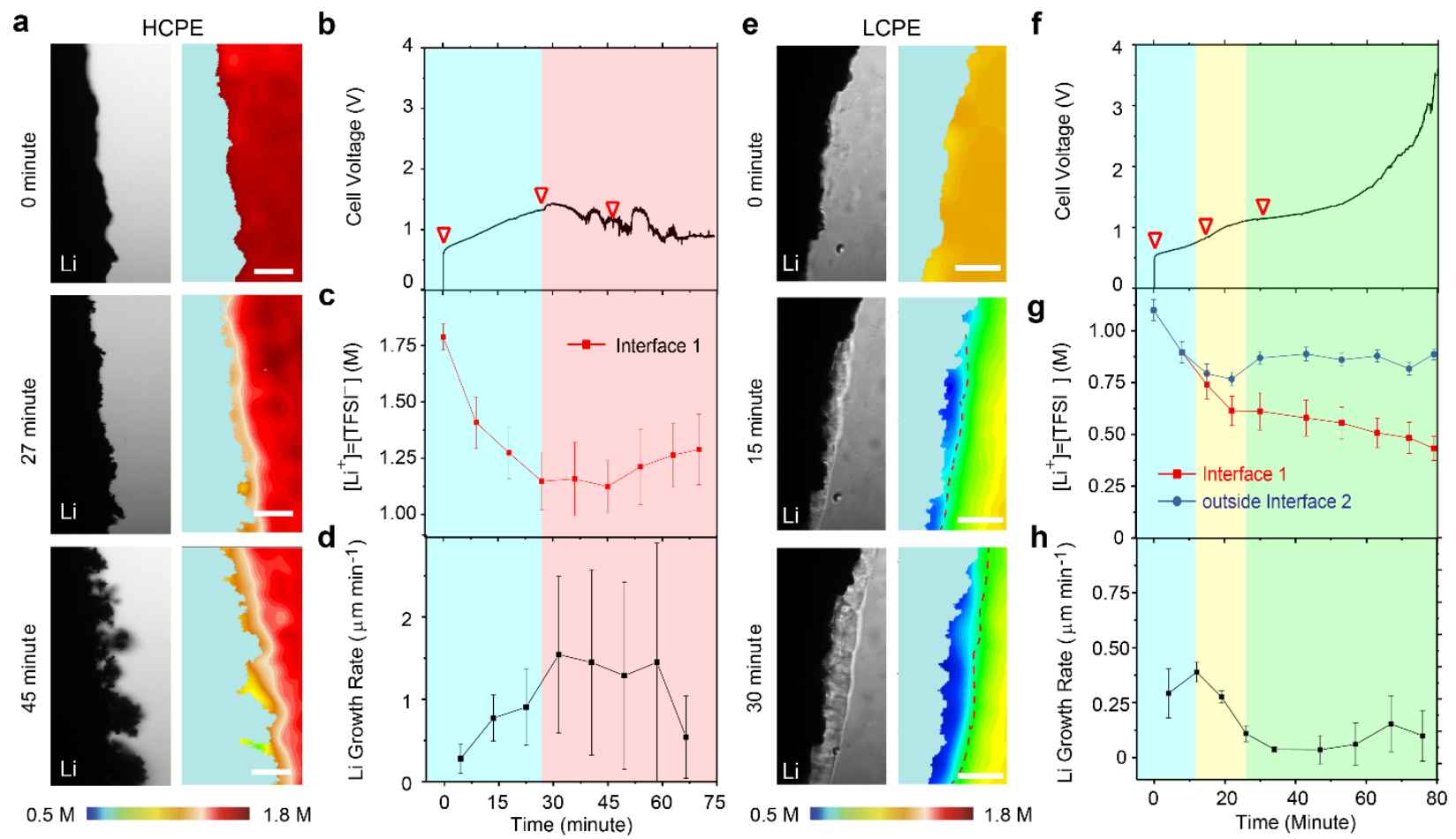

Fig. 2. Lithium growth at the lithium/PEO electrolyte interface with HCPE or LCPE. a-d, lithium dendrite growth in HCPE. a, the bright field and corresponding SRS images of $\left[\mathrm{Li}^{+}\right]=\left[\mathrm{TFSI}^{-}\right]$at three representative stages. $\mathbf{b}$, the voltage profile of the $\mathrm{Li} / \mathrm{Li}$ cell. $\mathbf{c},\left[\mathrm{Li}^{+}\right]=\left[\mathrm{TFSI}^{-}\right]$versus time, and $\mathbf{d}$, the lithium growth rate $(v)$ versus time. Interface 1 refers to the boundary between the lithium electrode and the PEO electrolyte. e-h, lithium growth in LCPE. e, the bright field and SRS images of $\left[\mathrm{Li}^{+}\right]$at three representative moments. $\mathbf{f}$, the voltage profile of the $\mathrm{Li} / \mathrm{Li}$ cell. The yellow shading corresponds to the appearance of the PEO-rich phase and the green shading indicates the regime where the PEO-rich phase has covered the entire lithium surface. $\mathbf{g},\left[\mathrm{Li}^{+}\right]=\left[\mathrm{TFSI}^{-}\right]$versus time, and $\mathbf{h}$, the lithium growth rate $(\boldsymbol{v})$ versus time. Interface 2 refers to the boundary between the new PEO-rich phase and the isotropic bulk polymer electrolyte, as marked by dash lines in SRS images in e. Scale bars are $50 \mu \mathrm{m}$. Zoom-in SRS images of a \& e are shown in Supplementary Fig. 4. 
In contrast to the conventional behaviors observed in HCPE, we find that ion depletion in LCPE induced a phase transformation process at the lithium/electrolyte interface that unexpectedly suppressed lithium dendrite growth, as observed by both SRS and bright-field (BF) images (Fig. 2e \& Supplementary Movie 2). First, the new phase appeared as the blue color region in SRS and granular-like region in $\mathrm{BF}$, which was confirmed by the spontaneous Raman spectrum (Supplementary Fig. 5). This new phase had a much lower [LiTFSI] than that in the isotropic bulk LCPE, as shown by the contrast between [LiTFSI] at the Li/electrolyte interface (interface 1), and [LiTFSI] outside the boundary between the new phase and the isotropic bulk electrolyte (interface 2). This difference increased from $0.79 \mathrm{M}$ vs. $0.74 \mathrm{M}$ at $\mathrm{t}=15 \mathrm{~min}$, to $0.87 \mathrm{M}$ vs. $0.61 \mathrm{M}$ at $\mathrm{t}=30$ min, and 0.89 M vs. 0.43 M at t= $79 \mathrm{~min}$ (Fig. 2g). Further study showed that the new phase was also poor in $\mathrm{SN}$, [SN] was only $\sim 1.8 \mathrm{M}$ at the interface, compared to $3.8 \mathrm{M}$ outside the interface 2 (Supplementary Fig. 6 and 7).

The appearance of this new phase unexpectedly but effectively suppressed the growth of lithium dendrites. Although the lithium dendrite growth was observed at $\mathrm{t}=0$ with $v$ of $\sim 0.3 \mu \mathrm{m}$ $\min ^{-1}$ (87\% porosity), $v$ quickly dropped to $0.048 \mu \mathrm{m} / \mathrm{min}$ at $\mathrm{t}=30 \mathrm{~min}$ after the initial formation of PEO-rich phase (Fig. 2h), equivalent to a porosity of 16\%. At this stage, the PEO-rich phase progressively formed on the lithium metal surface. After the new phase fully covered the Li metal surface, the average $v$ from $30 \mathrm{~min}$ to $63 \mathrm{~min}$ was only $0.044 \mu \mathrm{m} \mathrm{min}^{-1}$, which corresponded to a low porosity of $9.2 \%$, about one thirtieth of that in HCPE, indicating a dense and uniform lithium deposition. Such behavior is not only self-forming but also self-reinforcing during ion depletion. For example, if Li dendrite grows fast at a certain location, the local current density will increase and lead to faster ion depletion and hence thicker new PEO-rich phase, which in turn suppresses dendrite growth (Supplementary Movie 1, LCPE). This active protection mechanism is distinct from other conventional passive protective layers, which requires perfect uniformity and durability. This suppression mechanism has not been reported in the literature, to the best of the authors' knowledge. This dynamic phase transformation and dendrite suppression are illustrated in Supplementary Movie 2.

The suppression mechanism and underlying mechano-chemical coupling. To understand how the new PEO-rich phase formed and suppressed dendrite growth, we first constructed the ternary phase diagram of PEO-LiTFSI-SN with the assistance of SRS (Fig. 3a), as it could measure the 
composition of each phase in a high-throughput fashion. The phase diagram illustrates a singlephase isotropic zone (I-zone) in the middle (orange), and three two-phase regions at the corners (white \& contour region). Representative SRS images of each region are shown in Supplementary Fig. 8. As shown in Fig. 3a, the composition of HCPE is at the center of the I-zone. When a lowto-mid-level current is applied, it progressively reduces [LiTFSI] on the lithium metal surface (Path A in Fig. 3a). Due to the high salt concentration in HCPE, the electrolyte composition at the Li/electrolyte interface remains within the I-zone during ion depletion, and thus no phase transformation occurred. In contrast, the composition of LCPE is close to the boundary between the single-phase I-zone and the two-phase region, making it susceptible to undergoing a phase transformation in response to ion depletion (Path B in Fig. 3a), since forming two phases is thermodynamically more stable than staying in one phase. This phase transformation reduces not only [LiTFSI], but also [SN] in the new phase, as confirmed by the redistribution of [SN] during the phase transformation (Supplementary Movie 3 and Supplementary Fig. 6 \& 7).

a

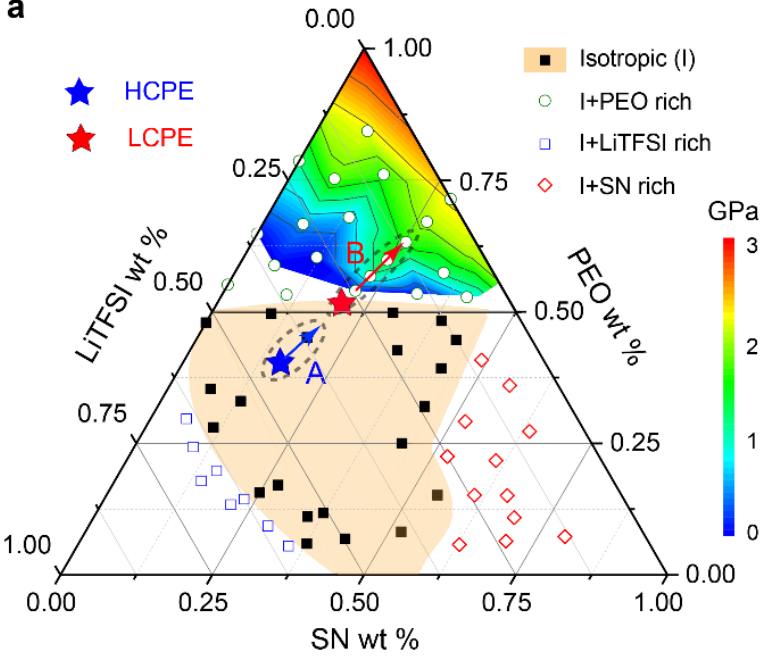

f

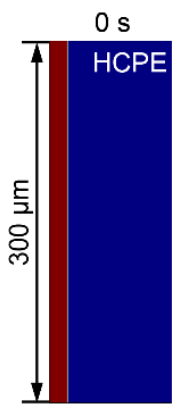

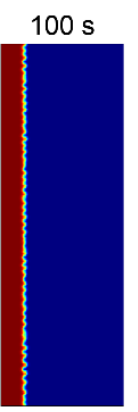

$200 \mathrm{~s}$

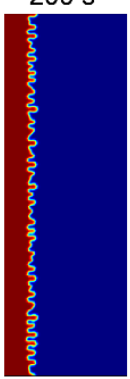

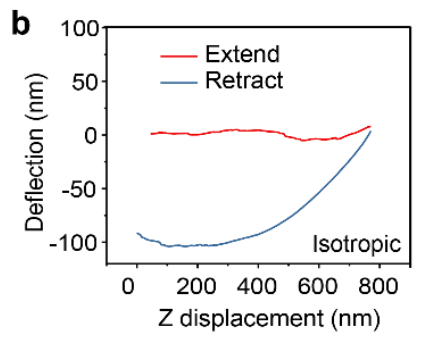

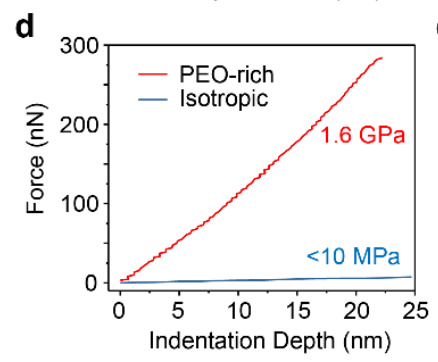

$400 \mathrm{~s}$

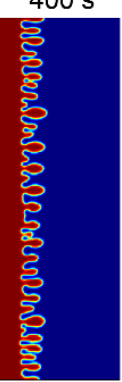

g

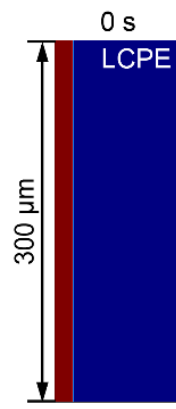

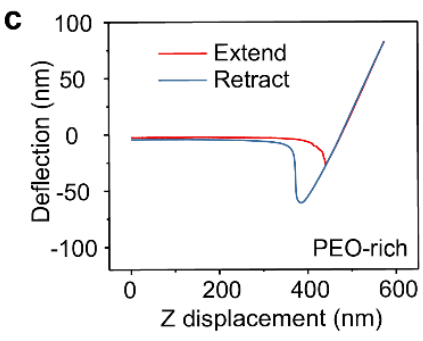

e

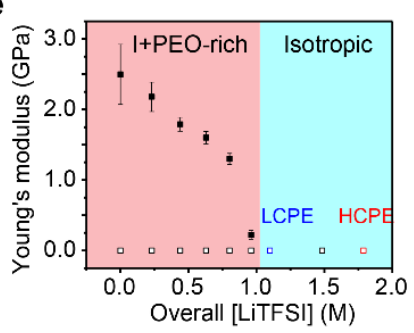

Fig. 3. The phase diagram and the mechanical properties of PEO-LiTFSI-SN polymer electrolytes. a, the ternary PEO-LiTFSI-SN phase diagram in weight percentage. The orange color marks the region of the 
isotropic single-phase region, while other regions represent two phases. The rainbow color at the top of the triangle is the contour of Young's moduli of as-formed PEO-rich phases at corresponding compositions in the phase diagram. $\mathbf{b} \& \mathbf{c}$, the AFM extending and retraction force curves of $\mathbf{b}$, the isotropic bulk phase (Izone, HCPE). No cantilever deformation is detected. $\mathbf{c}$, the as-formed PEO-rich phase with a composition of 0.52 M LiTFSI and 2.6 M SN (See supplementary Table 1 for details). d, the corresponding forceindentation curves for samples in $\mathbf{b}$ and $\mathbf{c}$. e, Young's moduli of the PEO-rich phase with different salt concentrations. The sample compositions are along the path A / B in a. All samples have the same SN/PEO ratio as HCPE and LCPE (40 wt\%), but different [LiTFSI]. The exact composition of each phase is listed in Supplementary Table 1. The solid and empty squares show Young's moduli of the PEO-rich phase and the isotropic bulk phases, respectively. $\mathbf{f}-\mathbf{g}$, phase-field simulations of Li electrodeposition in solid polymer electrolytes with $\mathbf{f}$, high concentration ( $2 \mathrm{M}$ salt) and $\mathbf{g}$, low concentration (1 M salt) at $0.5 \mathrm{~mA} \mathrm{~cm}{ }^{-2}$.

The effective suppression of Li dendrites by the new PEO-rich phase is hypothesized to arise from mechano-chemical coupling during the phase transformation at the Li/electrolyte interface. To verify this, AFM was used to measure the Young's moduli of solid polymer electrolytes (SPE) with different compositions, including both the I-zone and the PEO-rich phase, and the results are superimposed onto the phase diagram in Fig. 3a. For the composition of the HCPE (1.8 M LiTFSI and 2.4 M SN), the approaching curve showed no deformation of the cantilever when the tip was pressed into the electrolyte, indicating that the electrolyte was very soft (Fig. 3b). Large cantilever deflection is detected during tip retraction, confirming that the tip was pressed inside HCPE and the electrolyte was sticky. All SPEs within the I-zone and the isotropic phase of SPEs in the two-phase regions in Fig. 3a displayed similar results (Young's modulus $E<10 \mathrm{MPa}$ ), indicating they could not mechanically suppress lithium dendrite growth. Conversely, a classic force curve was observed for the PEO-rich phase formed in SPE. The sample had an overall composition of 0.6 M LiTFSI and 3.3 M SN, and the PEO-rich phase inside contained 0.52 M LiTFSI and 2.6 M SN (Fig. 3c). Using the Sneddon model, ${ }^{49}$ the corresponding $E$ was $1.6 \mathrm{GPa}$, well beyond the threshold to suppress lithium dendrites in previous studies, ${ }^{24}$ thus explaining why dendrite growth was suppressed in LCPE. The conductivity of the as-formed PEOrich state is in the order of $10^{-5} \mathrm{~S} \mathrm{~cm}^{-1}$, which still allows $\mathrm{Li}^{+}$to shuttle (Supplementary Fig. 9).

To further understand mechano-chemical coupling at the Li/electrolyte interface, the composition-dependent mechanical properties of the PEO-rich phases in PEO electrolytes were systematically measured by AFM. As shown in Fig. 3e, the modulus of the PEO-rich phase quickly 
rises to 1.2 and $1.8 \mathrm{GPa}$ when [LiTFSI] is reduced to 0.80 and $0.44 \mathrm{M}$ in the PEO-rich phase (see Supplementary Table 1 for exact compositions in PEO rich phase). Further contour on the Young's moduli of the PEO-rich phases in the I + PEO-rich two-phase region (Fig. 3a) shows that the modulus is typically above $1 \mathrm{GPa}$ when [LiTFSI] is less than $0.8 \mathrm{M}$. The contour indicates that the mechanism of ion depletion-induced stabilization of lithium deposition is effective in a wide range of electrolyte compositions.

The proposed suppression mechanism is also supported by phase-field simulations which take the mechano-chemical coupling into account (Supplementary Note 3 and Supplementary Movie 4). Since no new phase was formed in HCPE, lithium dendrites grew fast in the soft isotropic bulk polymer electrolyte ( $E=10 \mathrm{MPa}$, Fig. 3f \& Supplementary Fig. 10a). In contrast, the rigid PEO-rich phase was formed $(E=1.6 \mathrm{GPa})$ once $\left[\mathrm{Li}^{+}\right]$was depleted below $0.85 \mathrm{M}$, effectively suppressing lithium dendrite growth (Fig. 3g \& Supplementary Fig. 10b). The deposited lithium was largely uniform and the $\left[\mathrm{Li}^{+}\right]$heterogeneity was low on the lithium surface. These simulation results strongly agree with experimental observations, supporting the hypothesis that the formation of the PEO-rich phase with high Young's modulus suppresses dendrite growth.

Ion depletion-induced stabilization of $\mathrm{Li}$ anode in $\mathrm{Li} / \mathrm{Li}$ and $\mathrm{LFP} / \mathrm{PEO} / \mathrm{Li}$ cells. With the understanding that the formation of a mechanically rigid PEO-rich phase can stabilize lithium deposition, we further examined the effectiveness of this strategy in repeated cycles. First, as a proof-of-concept experiment, $0.5 \mathrm{~mA} \mathrm{~cm}^{-2}$ was applied to a Li/PEO-LCPE/Li symmetric cell with a deposition capacity of $0.25 \mathrm{mAh} \mathrm{cm}^{-2}$ for 20 cycles at room temperature. As observed with the optical microscope (Fig. 4a \& Supplementary Movie 5), no obvious lithium dendrites formed in the first deposition, and the lithium protrusions were frozen by the growing PEO-rich phase, leading to stable lithium deposition. During the lithium stripping, both the PEO-rich phase and lithium metal electrode shrank without forming any dead lithium. Upon cycling, the lithium surface moved forward slightly after 20 cycles, indicating that this suppression mechanism was effective upon multiple cycles. Further tests showed that such a reversible behavior could be achieved in a wide range of currents from 0.25 to $1 \mathrm{~mA} \mathrm{~cm}^{-2}$ (Supplementary Video 5). It should be noted that the PEO-rich phase sometimes did not fully disappear during lithium stripping, which is attributed to its disappearance being a kinetically slow process. In contrast, without the PEOrich phase, fast dendrite growth was observed within the first several cycles for $\mathrm{Li} / \mathrm{Li}$ cells with 
HCPE, forming large amounts of dead lithium during the repeated stripping process (Fig. 4 b \& Supplementary Movie 5).
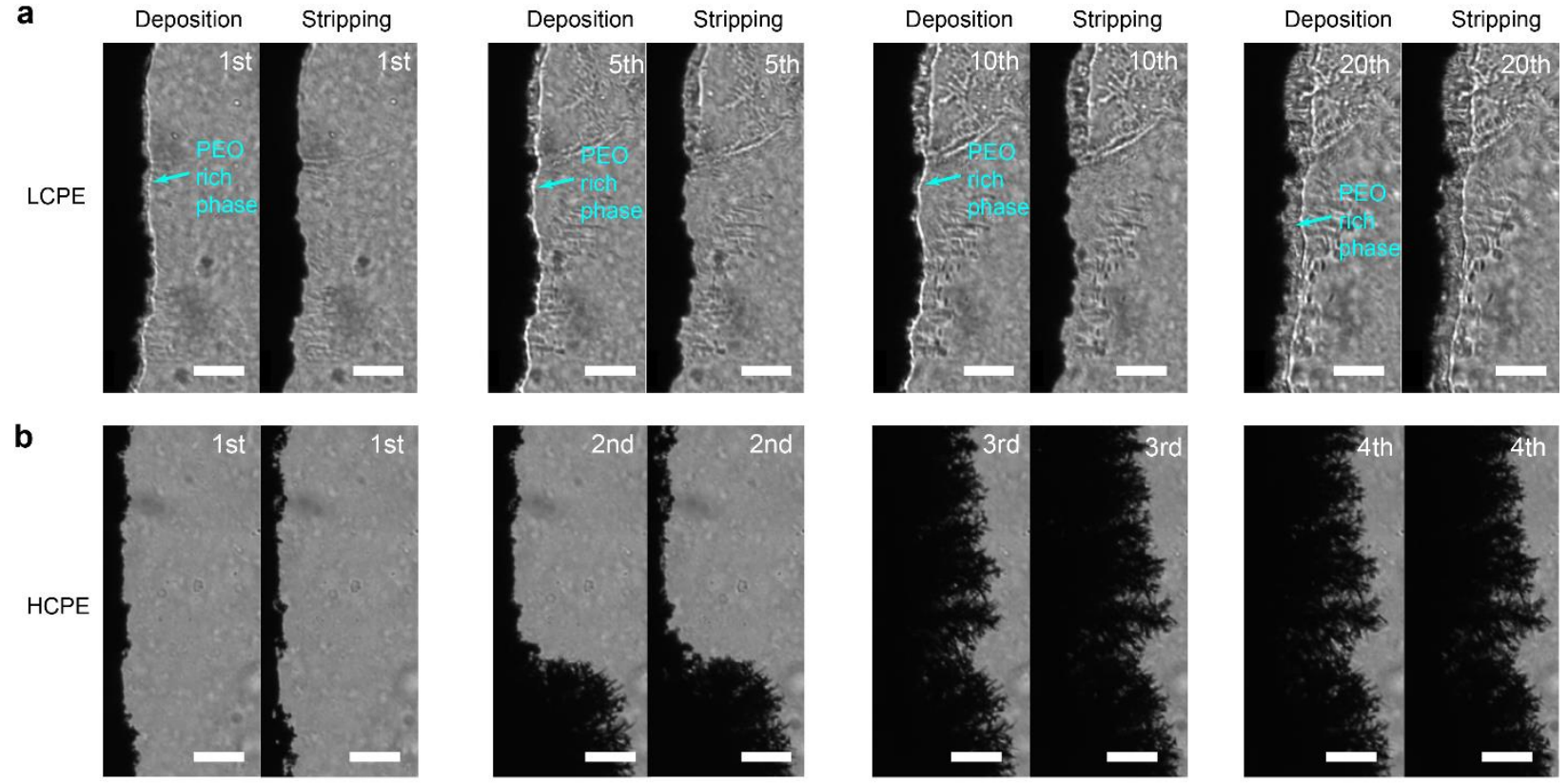

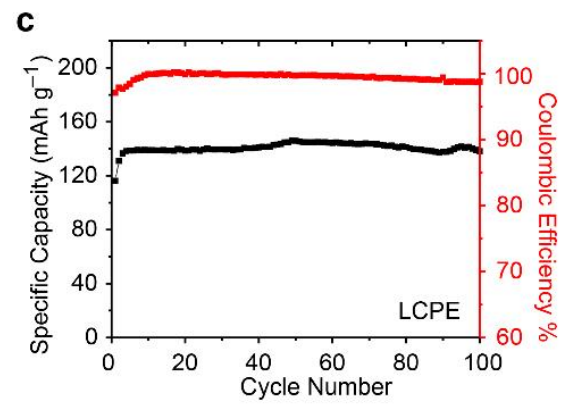

d
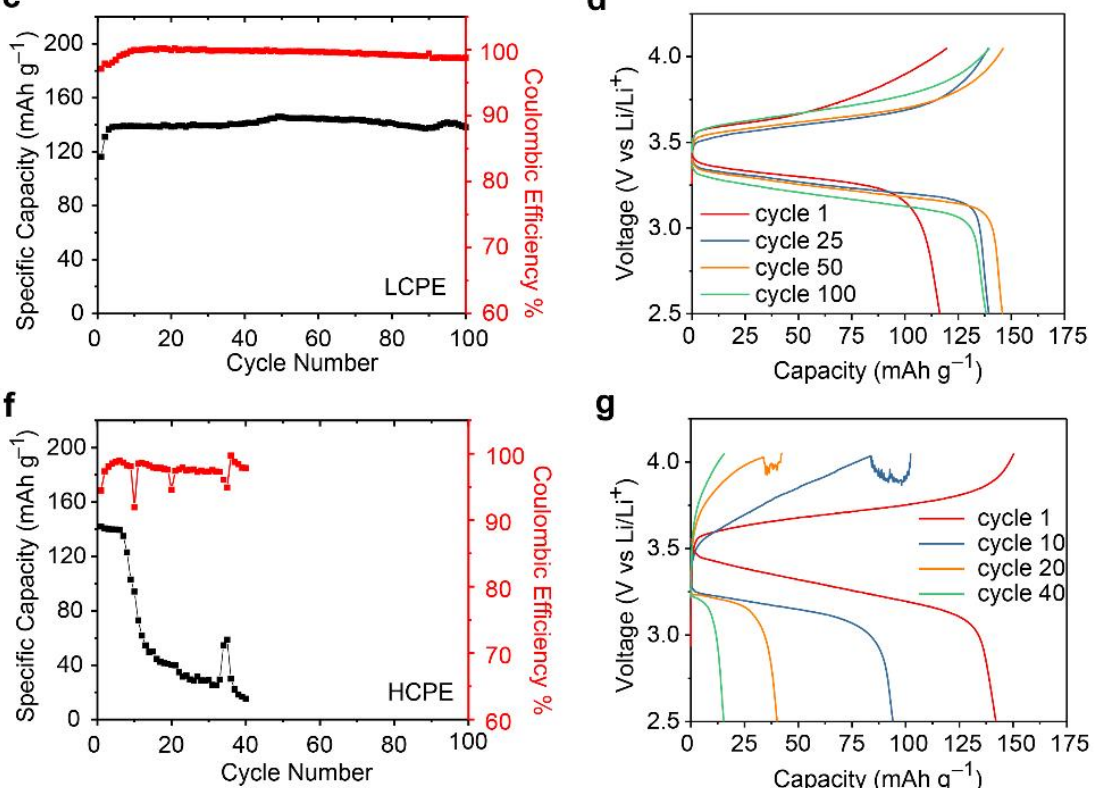

g
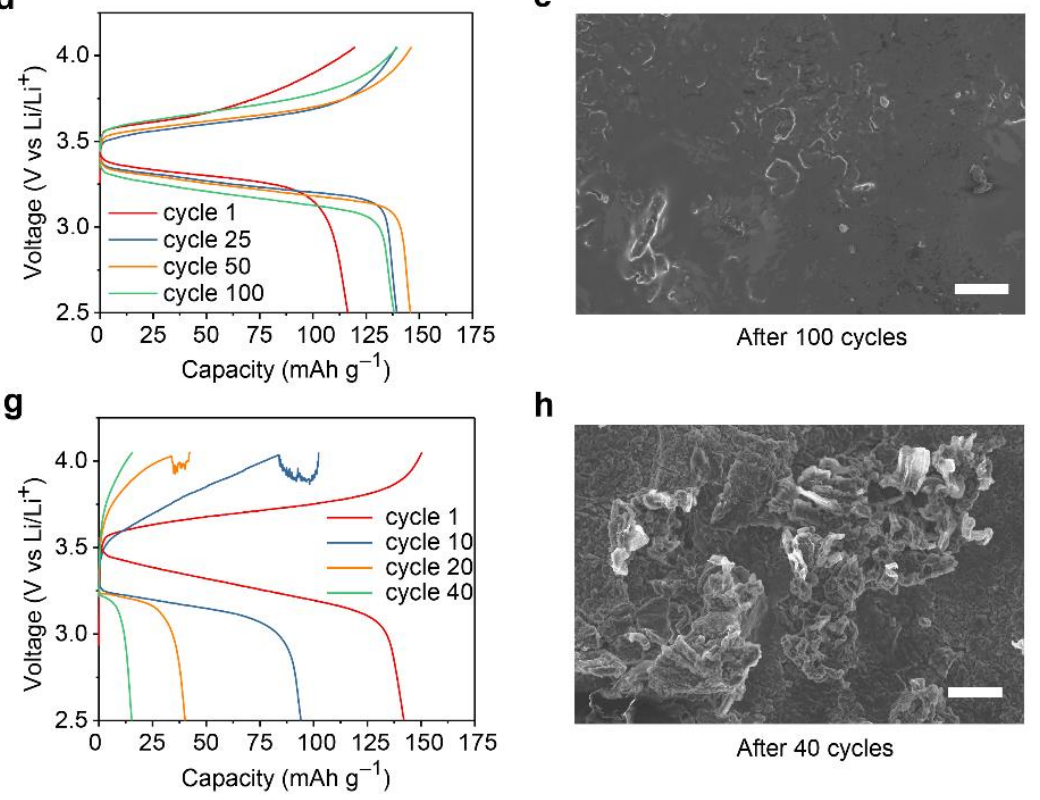

h

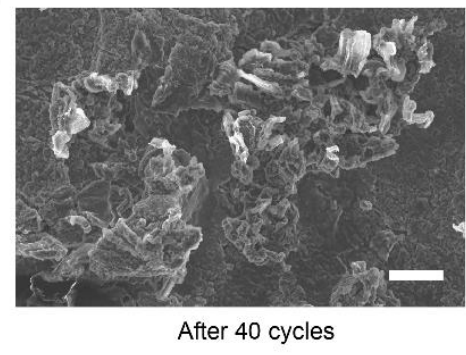

Fig 4. Cycling stability of the lithium metal anode in LCPE and HCPE. a-b, the bright-field images of the lithium electrodes during lithium plating and stripping in a. LCPE and $\mathbf{b}$. HCPE. The scale bars are 100 $\mu \mathrm{m}$. c, the cycling performance of $\mathrm{LiFePO}_{4} / \mathrm{Li}$ metal battery with $\mathrm{LCPE}$ and $\mathbf{d}$, the corresponding voltage profiles. e, An SEM image of the lithium metal surface after 100 cycles. f, the cycling of $\mathrm{LiFePO}_{4} / \mathrm{Li}$ metal 
battery with HCPE and $\mathbf{g}$, the corresponding voltage profiles. h, An SEM image of the lithium metal surface after 40 cycles. The scale bars in $\mathbf{e}$ and $\mathbf{h}$ are $10 \mu \mathrm{m}$.

The effectiveness of this strategy was further demonstrated in LFP $\left(\sim 4 \mathrm{mg} \mathrm{cm}^{-2}\right) / \mathrm{PEO} / \mathrm{Li}$ cells at $0.25 \mathrm{C}$ and $40{ }^{\circ} \mathrm{C}$. The PEO electrolyte was $100 \mu \mathrm{m}$-thick without a separator (Supplementary Fig. 11). Stable cycling was achieved with LCPE. The initial discharge capacity was $120 \mathrm{mAh} \mathrm{g}^{-1}$ and slowly increased to $141 \mathrm{mAh} \mathrm{g}^{-1}$ in the 4 th cycle due to activation. After 100 cycles, the capacity remained at $140 \mathrm{mAh} \mathrm{g}^{-1}$, corresponding to a retention of $99 \%$. The average CE for cycle 5-100 is $99.6 \%$ (Fig. 4c). The voltage profile shows that the internal resistance only increases slightly and there is no sign of dendrite-induced short-circuit (Fig. 4d). SEM further revealed that the lithium metal surface was relatively flat after 100 cycles, with occasionally island-like morphology, demonstrating the effectiveness of the PEO-rich phase in suppressing lithium dendrites (Fig. 4e). On the other hand, the LFP/Li cell with HCPE quickly failed with the capacity dropping from 143 to $50.7 \mathrm{mAh} \mathrm{g}^{-1}$ after only 14 cycles (Fig. 4f). The voltage profile showed drastically increased overpotential, and the unstable voltage curve in charging suggests possible dendrite growth and micro-shorting (Fig. 4g). The average CE is only $97.4 \%$, which probably arises from the prosperous growth of lithium dendrites, as validated by SEM imaging (Fig. 4h).
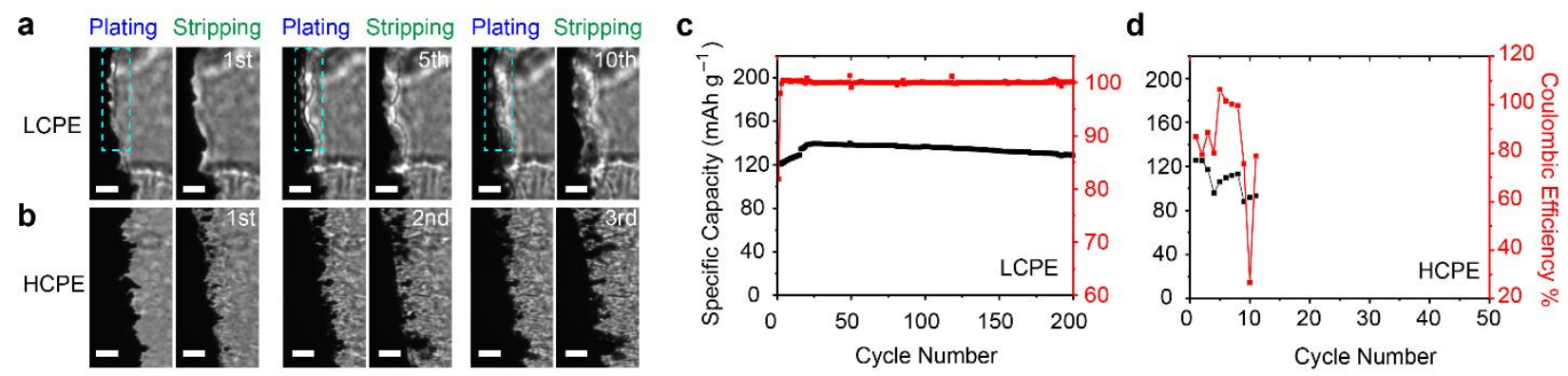

Fig 5. Cycling stability of the lithium metal anode in $\mathrm{Li} / \mathrm{Li}$ cells and $\mathrm{LFP} / \mathrm{Li}$ cells with PC/LiDFOB$\mathrm{LiBF}_{4}$ dual salt-based LCPE and HCPE. a/b, the bright-field images of the lithium electrodes in $\mathrm{Li} / \mathrm{Li}$ cells with (a) LCPE and (b) HCPE at room temperature and $0.75 \mathrm{~mA} \mathrm{~cm}^{-2}$. The scale bars are only $25 \mu \mathrm{m}$ to better show the as-formed new PEO-rich phase (The dashed cyanic area). c/d, the cycling performance of $\mathrm{LiFePO}_{4} / \mathrm{Li}$ metal batteries with (c) $\mathrm{LCPE}\left(\mathrm{EO} / \mathrm{Li}^{+}=6\right)$ and (d) $\mathrm{HCPE}\left(\mathrm{EO} / \mathrm{Li}^{+}=3\right)$ at $38^{\circ} \mathrm{C}$. The current density is $0.3 \mathrm{~mA} / \mathrm{cm}^{2}$. In both LCPE and HCPE, PC is used as the plasticizer and it is $90 \mathrm{wt} \%$ of PEO. 
Such phase separation-induced dendrite suppression is universal in PEO electrolytes. In another PEO electrolyte of lithium difluoro(oxalato)borate (LiDFOB) and lithium tetrafluoroborate $\left(\mathrm{LiBF}_{4}\right)$ as salts and PC as plasticizer, a similar phenomenon was also observed. In this system, phase separation exists when $\mathrm{EO} / \mathrm{Li}^{+}>6$ and it diminishes at $\mathrm{EO} / \mathrm{Li}=5.3$ (Supplementary Fig. 12), therefore, a PC-based LCPE with EO/ $\mathrm{Li}^{+}=6\left(2.9 \mathrm{mS} \mathrm{cm}{ }^{-1}\right)$ was tested in a $\mathrm{Li} / \mathrm{Li}$ cell at $0.75 \mathrm{~mA} \mathrm{~cm}^{-2}$. A PEO-rich layer was clearly observed during lithium deposition and capable of suppressing lithium dendrites (Fig. 5a and Supplementary Movie 6). In contrast, phase separation was not observed in the PC-based HCPE with EO/ $\mathrm{Li}^{+}=3\left(5.2 \mathrm{mS} \mathrm{cm}{ }^{-1}\right)$, and obvious dendrites and dead lithium were formed (Fig. 5b). The effectiveness of the strategy was also demonstrated in full cells. In an LFP/PC-based LCPE/Li cell with $5 \mathrm{mg} \mathrm{LFP} \mathrm{cm}^{-2}$ and $40 \mu \mathrm{m}$ thin lithium (Fig. 5c), the cell shows steady cycling over 200 cycles at $0.3 \mathrm{~mA} \mathrm{~cm}^{-2}(122,140$ and $129 \mathrm{mAh} \mathrm{g}^{-1}$ in cycle 1, 25 and 200, respectively). In contrast, when LCPE was replaced by HCPE, the cell dies after ten cycles due to dendrite-induced internal shorting (Fig. 5d). The corresponding voltage profiles are shown in Supplementary Fig. 13. Batteries with $10 \mathrm{mg} \mathrm{LFP} \mathrm{cm}{ }^{-2}(1.3 \mathrm{mAh}$ $\mathrm{cm}^{-2}$ ) and $40 \mu \mathrm{m}$ lithium also showed stable cycling. The cell capacity is $116 \mathrm{mAh} \mathrm{g}^{-1}$ in cycle 1 , $131 \mathrm{mAh} \mathrm{g}^{-1}$ in cycle 20 and $119 \mathrm{mAh} \mathrm{g}^{-1}$ in cycle 80 (Supplementary Fig. 14). Details of electrolyte composition and fabrication process can be found in Supplementary Note 1.

\section{Conclusion}

The dynamic ion depletion, phase transformation in polymer electrolytes, and their correlations with lithium deposition were observed for the first time thanks to the high chemical, temporal, and spatial resolutions of SRS microscopy. We successfully unveil phase transformation in the polymer electrolytes induced by ion depletion and the formation of a new PEO-rich phase at the electrode/electrolyte interface. This new phase has a high Young's modulus of up to $3 \mathrm{GPa}$, which is effective in mechanically suppressing lithium dendrite growth by functioning as a reversible, self-reinforcing protective layer on lithium anode. In contrast, without such phase transformation, conventional polymer electrolytes have a small modulus $<10 \mathrm{MPa}$, leading to fast lithium dendrite growth. This strategy is universal and effective with different salt and plasticizers. By utilizing this unexpected mechano-chemical coupling mechanism, we successfully demonstrated $\mathrm{LiFePO}_{4} / \mathrm{PEO} / \mathrm{Li}$ cells with 200 stable cycles, while cells without this mechanism failed quickly within 10 cycles. This study shows that phase transformation can be used as a new strategy to 
suppress lithium dendrite. This strategy is compatible with state-of-the-art battery materials and manufacturing processes without extra needs to control the conformability of protective layers in previous literature. It will facilitate the development of solid polymer electrolyte-based lithium metal batteries with enhanced thermal stability and high energy density.

\section{Acknowledgement}

We acknowledge seed funding support from Columbia University's Research Initiatives in Science \& Engineering (RISE) award, and Columbia SEAS Interdisciplinary Research Seed (SIRS) funding. Y. Y. acknowledge the support from the Air Force Office of Scientific Research (FA9550-18-1-0410). W. M. and Y. M. acknowledge the support from National Science Foundation (Grant No. 1904684). L. C. and Z. L. acknowledge the support from the Department of Energy, Office of Energy Efficiency and Renewable Energy (EERE), under the Award \#DEEE0007803 from the Battery Material Research (BMR) Program. X. C. and H. W. thank the support of the Office of Naval Research (ONR) under Grand No. N00014-18-1-2492. We also want to thank Prof. William Goddard at Caltech for his kind suggestions for this work.

\section{References}

1. Cheng, X.-B., Zhang, R., Zhao, C.-Z., Zhang, Q. Toward safe lithium metal anode in rechargeable batteries: a review. Chem. Rev. 117, 10403-10473 (2017).

2. Lin, D., Liu, Y., Cui, Y. Reviving the lithium metal anode for high-energy batteries. Nat. Nano. 12, 194 (2017).

3. Albertus, P., Babinec, S., Litzelman, S., Newman, A. Status and challenges in enabling the lithium metal electrode for high-energy and low-cost rechargeable batteries. Nat. Energy 3, 16-21 (2018).

4. Li, N. W., Yin, Y. X., Yang, C. P., Guo, Y. G. An artificial solid electrolyte interphase layer for stable lithium metal anodes. Adv. Mater. 28, 1853-1858 (2016).

5. Basile, A., Bhatt, A. I., O’Mullane, A. P. Stabilizing lithium metal using ionic liquids for longlived batteries. Nat. Commun. 7, 1-11 (2016).

6. Han, F., et al. High electronic conductivity as the origin of lithium dendrite formation within solid electrolytes. Nat. Energy 4, 187-196 (2019).

7. Wang, X., et al. Stress-driven lithium dendrite growth mechanism and dendrite mitigation by electroplating on soft substrates. Nat. Energy 3, 227-235 (2018).

8. Xiao, J. How lithium dendrites form in liquid batteries. Science 366, 426-427 (2019). 
9. Liu J, et al. Pathways for practical high-energy long-cycling lithium metal batteries. Nat. Energy 4, 180-186 (2019).

10. $\mathrm{Xu}, \mathrm{K}$. Nonaqueous liquid electrolytes for lithium-based rechargeable batteries. Chem. Rev. 104, 4303-4418 (2004).

11. Tikekar, M. D., Choudhury, S., Tu, Z., Archer, L. A. Design principles for electrolytes and interfaces for stable lithium-metal batteries. Nat. Energy 1, 16114 (2016).

12. Ren X, et al. Localized high-concentration sulfone electrolytes for high-efficiency lithium-metal batteries. Chem 4, 1877-1892 (2018).

13. Chen, S., et al. High-Voltage Lithium-Metal Batteries Enabled by Localized High-Concentration Electrolytes. Adv. Mater. 30, 1706102 (2018).

14. Lu, Q., et al. Dendrite-Free, High-Rate, Long-Life Lithium Metal Batteries with a 3D Cross-Linked Network Polymer Electrolyte. Adv. Mater. 29, 1604460 (2017).

15. Panday, A., et al. Effect of molecular weight and salt concentration on conductivity of block copolymer electrolytes. Macromolecules 42, $4632-4637$ (2009).

16. Stone, G., et al. Resolution of the modulus versus adhesion dilemma in solid polymer electrolytes for rechargeable lithium metal batteries. J. Electrochem. Soc. 159, A222-A227 (2012).

17. Hess, S., Wohlfahrt-Mehrens M, Wachtler M. Flammability of Li-ion battery electrolytes: flash point and self-extinguishing time measurements. J. Electrochem. Soc. 162, A3084-A3097 (2015).

18. Li, D., Fang, W., Xing, Y., Guo, Y., Lin, R. Effects of dimethyl or diethyl carbonate as an additive on volatility and flash point of an aviation fuel. J. Hazard. Mater. 161, 1193-1201 (2009).

19. Catoire, L., Naudet, V. A. unique equation to estimate flash points of selected pure liquids application to the correction of probably erroneous flash point values. J. Phys. Chem. Ref. Data 33, 1083-1111 (2004).

20. Li, S., et al. A superionic conductive, electrochemically stable dual-salt polymer electrolyte. Joule 2, 1838-1856 (2018).

21. Maitra, M. G., Sinha, M., Mukhopadhyay, A. K., Middya, T. R., De, U., Tarafdar, S. Ionconductivity and Young's modulus of the polymer electrolyte PEO-ammonium perchlorate. Solid State Ion. 178, 167-171 (2007).

22. Geng, H., et al. Fabrication and properties of composites of poly (ethylene oxide) and functionalized carbon nanotubes. Adv. Mater. 14, 1387-1390 (2002).

23. Klongkan, S., Pumchusak, J. Effects of nano alumina and plasticizers on morphology, ionic conductivity, thermal and mechanical properties of $\mathrm{PEO}-\mathrm{LiCF}_{3} \mathrm{SO}_{3}$ solid polymer electrolyte. Electrochim. Acta 161, 171-176 (2015).

24. Barai, P., Higa, K., Srinivasan, V. Lithium dendrite growth mechanisms in polymer electrolytes and prevention strategies. Phys. Chem. Chem. Phys. 19, 20493-20505 (2017).

25. Brissot, C., Rosso, M., Chazalviel, J. N., Lascaud, S. In Situ Concentration Cartography in the Neighborhood of Dendrites Growing in Lithium/Polymer-Electrolyte/Lithium Cells. $J$. Electrochem. Soc. 146, 4393-4400 (1999).

26. Liu, S., et al. Effect of nano-silica filler in polymer electrolyte on Li dendrite formation in Li / poly (ethylene oxide)-Li $\left(\mathrm{CF}_{3} \mathrm{SO}_{2}\right)_{2} \mathrm{~N} / \mathrm{Li}$. J. Power Sources 195, 6847-6853 (2010). 
27. Ramesh, S., Winie, T., Arof, A. Investigation of mechanical properties of polyvinyl chloridepolyethylene oxide (PVC-PEO) based polymer electrolytes for lithium polymer cells. Eur. Polym. J. 43, 1963-1968 (2007).

28. Fu, K. K., et al. Flexible, solid-state, ion-conducting membrane with 3D garnet nanofiber networks for lithium batteries. Proc. Natl. Acad. Sci. U. S. A. 113, 7094-7099 (2016).

29. Zeng, X., et al. Commercialization of lithium battery technologies for electric vehicles. Adv. Energy Mater. 9, 1900161 (2019).

30. Fang, C., et al. Quantifying inactive lithium in lithium metal batteries. Nature 572, 511-515 (2019).

31. Li, Y., et al. Atomic structure of sensitive battery materials and interfaces revealed by cryo-electron microscopy. Science 358, 506-510 (2017).

32. Zachman, M. J., Tu, Z., Choudhury, S., Archer, L. A., Kourkoutis L. F. Cryo-STEM mapping of solid-liquid interfaces and dendrites in lithium-metal batteries. Nature 560, 345 (2018).

33. Bhattacharyya, R., Key, B., Chen, H., Best, A. S., Hollenkamp, A. F., Grey, C. P. In situ NMR observation of the formation of metallic lithium microstructures in lithium batteries. Nat. Mater. $\mathbf{9}$, 504 (2010).

34. Chandrashekar, S., Trease, N. M., Chang, H. J., Du, L.-S., Grey, C. P., Jerschow, A.. ${ }^{7}$ Li MRI of Li batteries reveals location of microstructural lithium. Nat. Mater. 11, 311 (2012).

35. Zhang, L., et al. Lithium whisker growth and stress generation in an in situ atomic force microscope-environmental transmission electron microscope set-up. Nat. Nano., 1-5 (2020).

36. Chen, Y., et al. Li metal deposition and stripping in a solid-state battery via Coble creep. Nature 578, 251-255 (2020).

37. Yan, Y., Cheng, C., Zhang, L., Li, Y., Lu, J. Deciphering the Reaction Mechanism of LithiumSulfur Batteries by In Situ / Operando Synchrotron-Based Characterization Techniques. Adv. Energy Mater. 9, 1900148 (2019).

38. Cao, C., Shyam, B., Wang, J., Toney, M. F., Steinrück, H.-G.. Shedding X-ray Light on the Interfacial Electrochemistry of Silicon Anodes for Li-Ion Batteries. Acc. Chem. Res. 52, 2673-2683 (2019).

39. Yu, S.-H., Huang, X., Brock, J. D., Abruña, H.. Regulating key variables and visualizing lithium dendrite growth: an operando X-ray study. J. Am. Chem. Soc. 141, 8441-8449 (2019).

40. Cheng, Q., et al. Operando and three-dimensional visualization of anion depletion and lithium growth by stimulated Raman scattering microscopy. Nat. Commun. 9, 2942 (2018).

41. Fleury, G., et al. Resolving the Framework Position of Organic Structure-Directing Agents in Hierarchical Zeolites via Polarized Stimulated Raman Scattering. J. Phys. Chem. Lett. 9, 17781782 (2018).

42. Li, H., et al. Imaging Chemical Kinetics of Radical Polymerization with an Ultrafast Coherent Raman Microscope. Adv. Sci., 1903644 (2020).

43. Freudiger, C. W., et al. Label-free biomedical imaging with high sensitivity by stimulated Raman scattering microscopy. Science 322, 1857-1861 (2008).

44. Bai, P., Li, J., Brushett, F. R., Bazant, M. Z. Transition of lithium growth mechanisms in liquid electrolytes. Energy Environ. Sci. 9, 3221-3229 (2016). 
45. Alarco, P.-J., Abu-Lebdeh, Y., Abouimrane, A., Armand, M. The plastic-crystalline phase of succinonitrile as a universal matrix for solid-state ionic conductors. Nat Mater. 3, 476 (2004).

46. Brouillette, D., Irish, D. E., Taylor, N. J., Perron, G., Odziemkowski, M., Desnoyers, J. E. Stable solvates in solution of lithium bis(trifluoromethylsulfone) imide in glymes and other aprotic solvents: Phase diagrams, crystallography and Raman spectroscopy. Phys. Chem. Chem. Phys. 4, 6063-6071 (2002).

47. Fengler, O., Ruoff, A. Vibrational spectra of succinonitrile and its $\left[1,4-{ }^{13} \mathrm{C}_{2}\right]-,\left[2,2,3,3-{ }^{2} \mathrm{H}_{4}\right]$-and $\left[1,4-{ }^{13} \mathrm{C}_{2}-2,2,3,3-{ }^{2} \mathrm{H}_{4}\right]$-isotopomers and a force field of succinonitrile. Spectrochim. Acta A. 57, 105117 (2001).

48. Miyazawa, T., Fukushima, K., Ideguchi, Y. Molecular vibrations and structure of high polymers. III. Polarized infrared spectra, normal vibrations, and helical conformation of polyethylene glycol. J. Chem. Phys. 37, 2764-2776 (1962).

49. Sneddon IN. Boussinesq's problem for a rigid cone. In: Mathematical Proceedings of the Cambridge Philosophical Society. Cambridge University Press (1948). 